\title{
Bilateral lens subluxation through a crystalline lens ectopia in a girl of 7 years: what diagnosis
}

Jait Amina ${ }^{1 *}$, Leghmari Mina ${ }^{1}$, Jait Mohcine², Matsanga Olive Rosine ${ }^{1}$, Lezrek Omar ${ }^{1}$, Cherkaoui wafae ${ }^{1}$

${ }^{1}$ Ophthalmology A, Specialty Hospital, Rabat Souissi, Morocco

${ }^{2}$ Hygiene service Agourai Meknes, Morocco

Received: 11 September, 2016; Accepted: 14 November, 2016; Published: 24 November, 2016

*Corresponding author: Jait Amina, Ophthalmology A, Specialty Hospital, Rabat Souissi, Morocco, Tel no : +212658894613; Email: aminajait@gmail.com

\section{Abstract}

The ectopia lentis, primitive congenital malformation enters usually as part of a malformation syndrome (Marfan's syndrome, homocystinuria, Weill Marchesani's syndrome). The discovery of a case imposes an etiological investigation and family screening. The authors present a case of ectopia lentis in a girl of 7 years old in which the table of Marfan's syndrome was not complete, and the assay of homocysteine was normal. The authors evoke today the diagnosis of nonspecific conjunctival disease. The signs of Marfan's syndrome are emerging with age which justifies regular monitoring by ultrasound of the heart in search of a widening of the aortic root considered as a major sign of this pathology.

A 7 years old girl brought by his mother for a decreased visual acuity since 1 year. She's no out bred, last sibling of six, with no particular family history. She presents a stature advance (+ 2DS), a narrow elongated face, sunken eyes, arched palate and a dental overlap (Figure 1A). In the skeleton, the patient has a disproportion of members of the trunk and a lordosis (Figure 1B and 1C). Ophthalmologic examination finds a bilateral nasal superior subluxation with significant zonular stretching (Figure 2), we did not notice any degenerative lesion of retinal periphery. The corrected visual acuity was $1 / 10$ in both eyes. On the paraclinical level, a heart ultrasound showed minimal mitral insufficiency and tricuspid insufficiency. The assay of homocysteine was normal.

An anterior vitrectomy and phacophagie were performed. Visual rehabilitation was based on the use of glasses of aphakia and amblyopia treatment. Final visual acuity was 3/10 in both eyes with good academic recovery.

The ectopia lentis, primitive congenital malformation enters usually as part of a malformation syndrome (Marfan's syndrome, homocystinuria, Weill Marchesani's syndrome). The discovery of a case imposes an etiological investigation and family screening.

In our patient, as the table of Marfan's syndrome was not complete, and the assay of homocysteine was normal, today we evoke the diagnosis of nonspecific conjunctival disease. The signs of Marfan's syndrome are emerging with age which justifies regular monitoring by ultrasound of the heart in search of a widening of the aortic root considered as a major sign of this pathology.

A priority of the management of ectopia of the lens is to find and treat associated general illness that can be life-threatening in the long term.

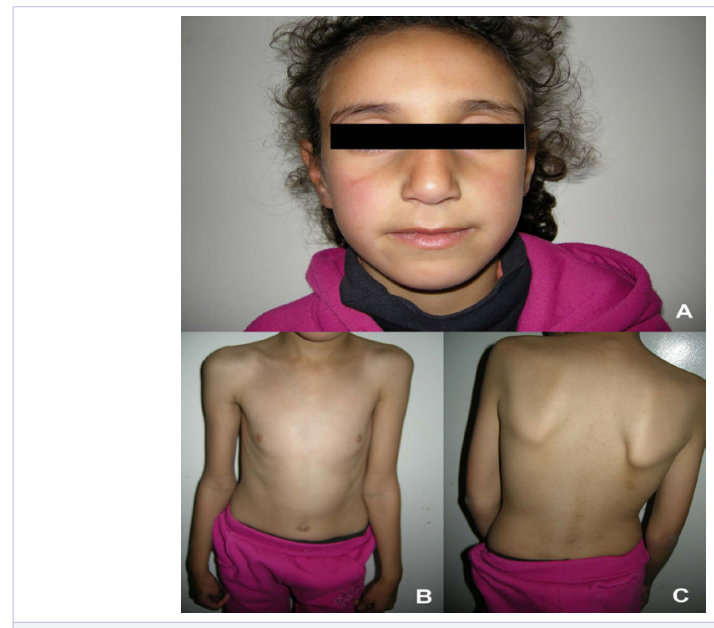

Figure 1

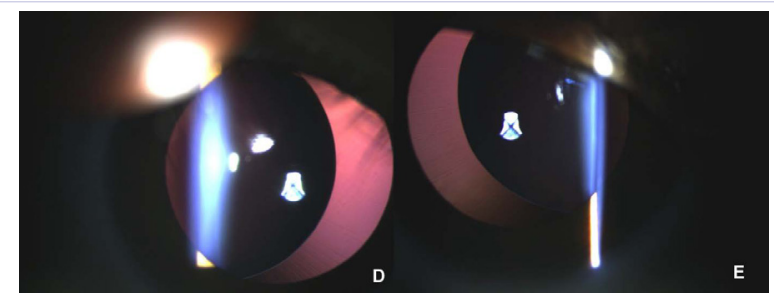

Figure 2 